This item was submitted to Loughborough's Research Repository by the author.

Items in Figshare are protected by copyright, with all rights reserved, unless otherwise indicated.

\title{
The use of freelisting to elicit stakeholder understanding of the benefits sought from healthcare buildings
}

PLEASE CITE THE PUBLISHED VERSION

http://dx.doi.org/10.1080/01446193.2012.658824

PUBLISHER

(C) Taylor and Francis

VERSION

AM (Accepted Manuscript)

LICENCE

CC BY-NC-ND 4.0

\section{REPOSITORY RECORD}

Thomson, Derek S., Ammar P.F. Kaka, Laura Pronk, and Chaham Alalouch. 2019. "The Use of Freelisting to Elicit Stakeholder Understanding of the Benefits Sought from Healthcare Buildings". figshare.

https://hdl.handle.net/2134/9668. 
This item was submitted to Loughborough's Institutional Repository (https://dspace.lboro.ac.uk/) by the author and is made available under the following Creative Commons Licence conditions.

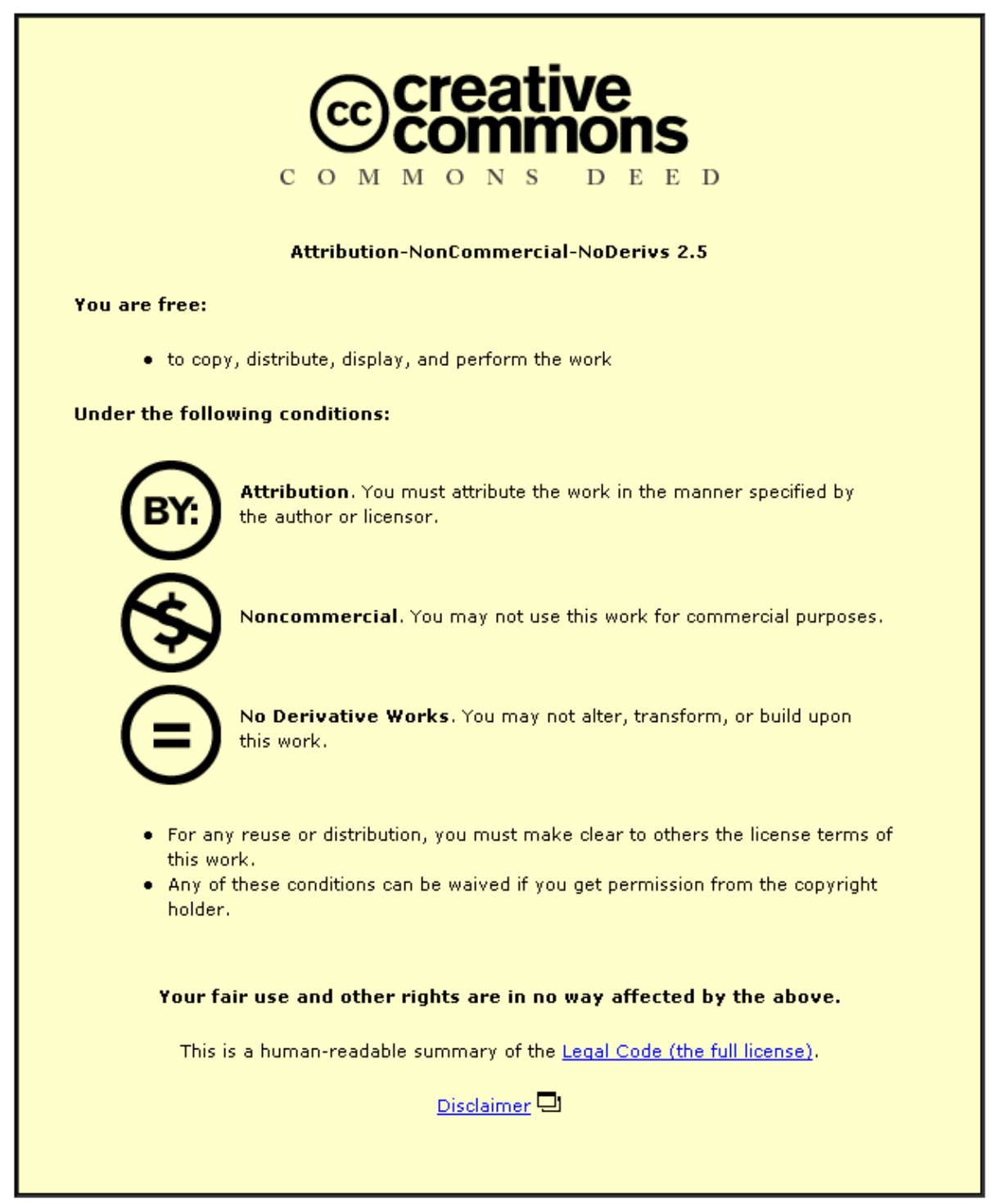

For the full text of this licence, please go to: http://creativecommons.org/licenses/by-nc-nd/2.5/ 


\title{
The Use of Freelisting to Elicit Stakeholder Understanding of the Benefits Sought from Healthcare Buildings
}

Thomson, D., Kaka, A., Pronk, L., Alalouch, C.

\begin{abstract}
Elicitation and synthesis of the collective understanding of a cultural domain held by a group of stakeholders is challenging. This problem typifies the pre-project activity from which a coherent understanding of the benefits sought from infrastructure investment must emerge to inform the business case rationale. The anthropological freelisting method is evaluated as a solution by determining its ability to be operationalised in a practical form for project application. Using data from the stakeholders of a large NHSScotland building project, the use of multidimensional scaling for data analysis is compared with participatory pilesorting to determine which freelisting protocol balances insight with practicality. Neither approach is found to offer an ideal method of characterising sought benefits. The social construction of pilesorting promotes reliability while the analytical rigour of multidimensional scaling remains attractive to auditors. Their distinct insights suggests that both approaches should be combined in future and used alongside further post-elicitation devices from anthropology such as cultural consensus modelling or structured conceptualisation.
\end{abstract}

\section{Keywords}

Construction management;Freelisting; Health care; Stakeholder; Benefits realisation

\section{Introduction}

At the turn of the century, UK government policy advanced the notion that "good [building] design provides a host of benefits" (Department for Culture 2000: 1). Since then, public sector building projects have been required to define the benefits - the desired traits of their stakeholders' situation brought about by the project - they intend to offer and to demonstrate benefit realisation upon building use (The Scottish Government 2009a). Despite this need, proven methods of eliciting stakeholder understanding of benefits do not yet exist. This study searches for a benefits elicitation method that balances analytical rigour with the need for a workable approach suited to practical construction project settings where contact time with stakeholders, and their willingness to engage in workshop activities, is often limited. The anthropological technique of 'freelisting' is examined because, when coupled with an appropriate analytical technique, it has a proven ability to elicit and synthesise a coherent view of collective 
understanding of a cultural domain: 'benefits' in this case. It is necessary to evaluate the data analysis that must be coupled to this elicitation method to complete the modelling protocol. Two approaches to synthesising insight from 'free' lists elicited by nonspecific prompt are evaluated. One uses participatory pilesorting, while the other uses multidimensional scaling for data reduction. Both protocols adapt the generalised freelisting protocol consistently encountered in the anthropological study design literature to operationalise the technique for application. The reported study sought to characterise the 'usefulness' of either protocol by considering the soundness of the insights it yielded in comparison with its attendant analytical complexity. The discussion that follows presents the comparison of both techniques structured by considering the differing insight they yielded and the extent to which they stimulated social processes between stakeholders from which complementary understanding of benefits to that represented in the model itself may have developed.

\section{Origins of Benefits Realisation Management}

In the UK, creators of public sector infrastructure such as buildings are constrained by government policy. This is closely associated with a 'value agenda' (typified by CABE 2006 and The Scottish Government 2009b) in which the relationship of users to buildings is carefully considered and principles such as evidence based design are promoted. This broadens expectations of asset performance beyond functionality, build quality, and cost to embrace the relationship of buildings to their stakeholders, including - in the healthcare sector - user experience of receiving care within them. More generally, UK central government policy attempts to ensure public infrastructure provides 'benefits' to stakeholders; key of which are benefits arising from what users consider good design to be. While much debate has focused on the tenets of design quality (Gann 2003), the benefits of good design are seldom evaluated in investment governance, despite the healthcare sector's growing appreciation of the 'evidencebase' explaining the role of good building design in promoting patient recovery (Mills et al. 2010, Codinhoto et al. 2008, Ulirch et al. 2008).. The Managing Successful Programmes guidance (MSP and Office of Government Commerce 2007) advises a Benefits Realisation Management Process (BRMP) to oversee benefits realisation throughout asset life. Such a BRMP creates a delivery process in which benefits realisation must be measured (either directly or by using proxy metrics for intangible benefits) but it does not provide a way of eliciting the benefits to be realised. Even healthcare-specific BRMPs, such BeReal (Sapountzis et al. 2009), do not yet resolve this critical task. As benefits are perceived and experienced by stakeholders and therefore have to be defined from their perspective, there is a pressing need to develop project-level stakeholder engagement methods from which commonly sought project benefits can be identified so that investment activity focuses on their realisation.

The development of a benefits elicitation method requires understanding of the nature of investment project benefits per se. Generally, a benefit is a desired outcome from change. Investments in healthcare buildings, like any other investment advancing a strategy, must bring about an end-state that reflects terminal (Rokeach 1973) stakeholder values. This grounding in values requires benefits realisation to be judged rather than measured. The majority of the benefits sought from healthcare buildings follow evidence-based design principles and are intangible in nature. Adbul-Samad and Macmillan (2005: 898) note that intangible benefits are 
situation-dependent and "a matter of value judgements and [are] subjective." They also suggest that 'value' results from the delivery of benefits for which clients are willing to pay. Thomson et al. (2003) note that stakeholders perceive value by trading-off the benefits they seek from a building investment against the sacrifices they have to make to gain those benefits. Indeed, Thomson et al. (2010) have opined that the 'willingness to pay' stated preference valuation technique advocated by HM Treasury (2003) and Pearce and Özdemiroglu (2002) could be used to quantify the realisation of both tangible and intangible benefits, thereby informing the performance measurement component of a BRMP. Methods of defining the project benefits to be realised and quantified remain unaddressed.

Benefits do not, as Rooke et al. (2010) suggest, equate directly with value when informing a BRMP (cf. Thomson et al. 2003). They are distinct investment decision criteria (Ratneshwar et al. 1997) that describe those attributes of goods that cause customers to perceive value (Sweeney and Soutar 2001) and relate it to price (Hamilton 2002). Because values frame these judgements, benefits are uniquely understood by each stakeholder. The creation of a single project solution, however, necessitates stakeholder negotiation of a unifying definition that, when realised, will yield a satisficing (Simon 1957) outcome. This need for synthesis is unique to project benefits elicitation and distinct from project value, which is always judged individually by each stakeholder in light of their individual values. Reiss et al. (2006) suggest that a benefit elicitation process should ideally also develop common understanding among stakeholders.

Diversity in stakeholder understanding of sought benefits is problematic to investment project managers and building designers. For example, healthcare stakeholders such as clinicians and nursing staff often consider benefits to result from healthcare consumption across socioeconomic groups and may consider them in solely monetary terms (McIntyre and Ataguba 2011). They may also consider benefits in a more amorphous form; resulting from the faith placed by the populace in the utility and fairness of their health systems (Block et al. 2001). Stakeholders with a construction background such as architects and project managers tend to have a more instrumental view and consider "benefit management [to be] increasingly recognized as a key formal activity within program management" (Morris and Jamieson 2004). The desired effect of benefits must be defined (Remenyi and Sherwood-Smith 1998) so that stakeholders' understanding of those benefits can direct the design, construction and use of the buildings being created.

Although policy requires the business case underpinning each healthcare building to define sought benefits, Thomson et al. (2010) have established that these definitions seldom reflect the benefits sought by stakeholders. Instead, business case expressions of benefits tend to reflect the funding body's investment programme intent or policy rather than project-specific issues. This occurs as, in current practice, the business case is primarily used to secure funding rather than to meaningfully explore project intent. Further, the imposition of programme benefits onto projects often precludes the social construction of stakeholder agreement about sought benefits and reduces the likelihood of stakeholders supporting any decreed to a project by an investing authority. A field based, project-specific elicitation of sought benefits is therefore required. 


\section{The Benefits Elicitation Problem \\ The Context of Benefits Elicitation}

The benefits sought from investments in NHS buildings are currently elicited using ad hoc arrangements of interviews, surveys, or the scoring of generic benefits by project relevance. Benefits elicitation currently commences in business case development and, although stakeholders are routinely engaged in that activity, the insight yielded is variable due to inconsistency in the methods used and their possible limited efficacy. A systematic benefits elicitation method is required which: gives each stakeholder equal opportunity to contribute; sustains engagement by providing an immediate outcome; builds a common understanding of project intent among stakeholders; and establishes an audit trail.

\section{Requirements of a Benefits Elicitation Method}

The elicitation of benefits requires two distinct activities. First, all stakeholders must be guided through an elicitation process to have an opportunity to express their individual view of what the project benefits could be. Second, these disparate views must be synthesised into a coherent definition of project benefits, ideally using an approach that builds common stakeholder understanding and buy-in to that definition.

\section{Freelisting}

When deployed at a project level, the benefits elicitation process must be quick and effective. The process must rapidly capture a large number of possible project benefits for analysis to determine which should be included in a common definition. The 'freelisting' technique commonly used by cultural anthropologists is ideally suited to this task as the stakeholder population can be readily surveyed and the project itself provides a convenient boundary to the 'project benefits' cultural domain studied. Freelisting offers notable advantages over the alternative group interviews, focus groups, task observation, and so forth in which the dynamics of such group situations are complex and a facilitator's ability to address them in practical settings cannot be guaranteed. Freelisting avoids these issues by eliciting stakeholder understanding consistently. Its tightly structured elicitation process ensures parity between participant stakeholders by affording each an equal role in the contribution of views. It allows each participant to fully express their individual understanding of the domain without being exposed to the bias of other participants. Used to structure elicitation in a workshop, it negates the need for facilitator moderation of group dynamics by compensating for emergent participant power distances, temporary and dynamic coalitions, and possible groupthink although, as will be seen, these demands may continue to be placed on the facilitator by the choice of subsequent synthesis activity, potentially creating inherent issues of analysis reliability.

\section{The Principles of Freelisting}

'Freelisting' is a "deceptively simple, yet powerful technique" (Bernard 2006: 301) used by cultural anthropologists to model how groups collectively understand a cultural domain: "a symbolic category described by a cover term" (Schrauf and Sanchez 2008: S385) which, in this application, is 'project benefits.' Its applications are diverse and growing, including: how inmates perceive the discipline regime of their prison (Fleisher and Harrington 2009); how office workers socially classify each other in formal organisations (Brewer 1995a); and how consumers interpret brands 
as a result of marketing (Ares and Deliza 2010). With specific reference to benefits, the technique has externalised "the enduring readiness with which specific benefits are brought to mind in relation to a given product category" (Ratneshwar et al. 1997: 247) to inform product marketing.

Although not espoused by a specific author, a generalised protocol is evident in the literature (Figure 1). This protocol elicits a 'freelist' from each stakeholder describing their individual understanding of the cultural domain under study. The terms on these lists are combined by their salience within the collective understanding of a group of stakeholders and forwarded to analysis to expose commonalities in stakeholders' collective understanding of that cultural domain.

\section{FIGURE 1 ABOUT HERE}

The elicited freelists comprise lists of 'terms:' single words the stakeholder associated with the cultural domain. Stakeholders are directed to simply list "what comes to mind" (Schmitt 1998: 319) in respect of an initial prompt without constraint or filter: hence 'free' list. The technique is readily opererationalised as stakeholders see it as simple word association, however careful design of the elicitation process ensures stakeholders' cognition of the cultural domain is accurately captured. This relies on communicating the expectation that stakeholders will express a large number of terms in a short time period (Bousfield and Barlcay 1950).

Following the generalised protocol, all the elicited lists are considered together and the 'salience' of each term within stakeholders' collective understanding of the cultural domain is calculated. Smith (1993), Sutrop (2001), and Smith and Borgatti (2010) independently define a 'salience index' to represent the commonality of term rank position and frequency across each stakeholder's freelist. Those terms most commonly and prominently associated with the cultural domain under study are the most salient and are forwarded to cluster analysis under the protocol.

In the generalised protocol, cluster analysis finds cognitively associated terms. Clusters of associated terms represent a thematic element of stakeholders' collective understanding. In this application, they represent the benefits sought from the building. Two approaches to cluster identification can be used. A statistical approach interrogates the salience indices of elicited terms to cluster of those of similar prominence within the collective cognition. Multidimensional scaling (MDS) identifies clusters by analysing the Euclidean distances between salience indices. An alternative participatory approach engages stakeholders in grouping and sorting to identify thematically-consistent groups (Blake et al. 2007) by forming clusters of associated terms that make sense (Ensign and Gittelsohn 1998) to them. The former approach replaces the stakeholder sense-making from which common understanding is socially constructed (Thiry 2001, Maitlis 2005) with statistical analysis. The anthropological freelisting literature advocates analytical, rather than participatory, approaches, whereas the building design literature favours socially-orientated participatory approaches (Jenkins and Forsyth 2010). 


\section{Obstacles to Operationalising the Generalised Protocol in Construction Projects Practical Considerations}

Barriers exist to application of the generalised freelisting protocol. If freelisting is to be accepted by healthcare building projects, the need to rigorously identify thematic project benefits must be balanced with the need for quick and simple engagement. Analytical integrity may therefore have to be compromised to operationalise the protocol while retaining sufficient rigour to produce a sound definition of project benefits for address by a BRMP.

Recalling Figure 1, the generalised protocol requires the analyst to harmonise language across a set of freelists. This removal of 'noise' from the dataset is one of the most complex and readily criticised aspects of freelisting. When single words and short phrases are elicited, codification reduces list complexity using synonyms and by exchanging morphological derivates (i.e. sad; sadness) to represent terms (Schrauf and Sanchez 2008). Codification becomes increasingly subjective as term expressions become more complex as it becomes necessary for the analyst to infer term meaning. This is particularly problematic when stakeholders, counter to direction, express terms in more complex forms than single words. Codification akin to that of content analysis, with its attendant bias risk, becomes necessary. To avoid the risk of introducing too much variability into the dataset for the analyst to attempt to infer meaning, all elicited terms, no matter what their form of expression, can be forwarded to cluster analysis.

Alternatively, stakeholders can be engaged in verification and harmonisation that combine terms considered to be related, thereby ensuring the thematic (dis)similarity of retained terms prior to the synthesis stage. Weller (2007: 344) proposes the building of a "cultural consensus model." This requires further post-freelisting data gathering from stakeholders by questionnaire or interview and a notable sample size; dependent upon the consistencies identified but suggested to be around 28 participants to provide 'validity' at the 0.95 level. This extent of stakeholder engagement would, it is posited, be impractical in 'live' construction projects where access to stakeholders is typically constrained and their willingness to participate in such activities would be compromised by the 'workshop fatigue' that causes repeated workshops to become progressively less insightful. Keeping freelisting in its generalised form (i.e. without Weller's consensus model) offers a simpler approach more appropriate to the limited resources that clients typically allocate to benefits realisation management, even though Weller's approach would assist in the social construction of consensus among stakeholders. Adaptation of the generalised form is still, however, required to ensure workability within the constraints of the typical construction project.

\section{Modification of the Generalised Protocol}

Difficulties in operationalising the generalised protocol in construction settings coupled with the need for a simple yet still sufficiently rigorous method, together with the need to minimise the risk of introducing the analyst's bias caused two modifications of the generalised protocol (Figure 2): an 'adapted' protocol (A') and an 'alternative' protocol (B) to be proposed. 


\section{FIGURE 2 ABOUT HERE}

The adapted protocol ( $\left.A^{\prime}\right)$ acknowledges difficulties in eliciting project benefits in live situations rather than the abstract experimental settings in which freelisting is typically deployed. As discussed, terms representing possible project benefits are often expressed by stakeholders as phrases or even short sentences rather than the preferred single words. This richness and specificity of term expression limits the analyst's ability to infer synonymic meanings as doing so would, potentially, introduce untenable bias. The analyst's ability to harmonise terms is thus constrained. The calculation of salience indexes on the noisy data elicited in practice would result in a scree plot with no clear distinction between salient terms and scree, in turn preventing data reduction prior to the MDS of the generalised protocol. The adapted protocol ( $A^{\prime}$ ) avoids this. After an initial harmonisation to combine those terms expressed as single words and for which synonyms can be readily identified without bias, all the remaining 'noisy' data is forwarded to MDS without reduction. The absence of salience indexes prevents compilation of a matrix of Euclidean term distances. The data is therefore prepared for MDS by considering the similarity of term expressions to compile a Boolean similarity matrix for processing. Compilation of this matrix arguably introduces the analyst bias that justified the removal of salience analysis. The consequences of this are considered below.

The alternative protocol (B) entirely removes the analyst's inference from data reduction and clustering by combining these activities and using the stakeholders to perform them in a participatory manner. It has the potential to yield meaningful and supported insight as stakeholders are presented with sense-making opportunities to build collective understanding. The resulting insight largely depends on a workshop facilitator's ability to fully engage every stakeholder in a 'pilesorting' activity while directing the social construction of common understanding of discussed terms. This can be considered a participatory, rather than mathematical approach to building Weller's consensus model.

\section{The Research Questions}

Two modified freelisting protocols have been proposed to address the practicalities of operationalisation in construction projects. These modifications raise two research questions:

RQ1: Do the adapted protocol ( $A^{\prime}$ ) and the alternative protocol (B) yield analogous results? $\mathrm{RQ} 2$ (conditional on RQ1): If $\mathrm{A}^{\prime}$ and $\mathrm{B}$ yield different results, which results are the most valid?

The research questions were explored by comparing application of protocol $\mathrm{A}^{\prime}$ and protocol B to the same freelist dataset.

Freelists were elicited from the stakeholders of a large NHSScotland capital investment project that had received funding and had commenced detailed design. A stakeholder workshop explored the viability of protocols $A^{\prime}$ and B with eight stakeholders, comprising: patient and staff representatives; NHS capital project members; estate management staff; and the project architect. These stakeholders were selected by the procuring NHSScotland Board to be 
representative of new facility users and operators. Most were represented by a senior member of their community and provided a sample of size and composition typical of that engaged in construction projects in benefits realisation (and, indeed, often in value management and design review activities). Schrauf and Sanchez (2010) confirmed that, when considered together, large sample sizes identify more terms, with $74 \%$ of the "topmost ten" (p. 83) terms being elicited from a group size of 20. This perspective on the freelisting activity is, however, predicated on the assumption that the cultural domain is finite. It is posited by the authors that the domain of 'project benefits' in construction is sufficiently amorphous that the likelihood of it being fully incorporated into stakeholders' cognition is low. The purpose of the freelisting activity forwarded here is, therefore, to inform benefits realisation. The benefits elicited are not required to be exhaustive but, instead, merely require those most salient among the stakeholders.

The workshop adopted the format and facilitation style typical of VM interventions. A facilitator skilled in the elicitation methods under study directed the workshop and was distinct from the stakeholders. Although an external facilitator would not interpret the project from the same perspective as the stakeholders (as Luck (2010) and Fleisher and Harrington (2009) recommend for reliability), the use of such a facilitator reflected anticipated benefits elicitation practice. The workshop format therefore realistically informed the test of the two proposed protocols; $A^{\prime}$ and $B$.

\section{Freelist Analysis}

\section{Freelist Elicitation}

To elicit freelists in a format facilitating both the adapted (A') and alternative (B) protocols, Bousfield and Barclay's (1950) freelisting method was modified. The constrained time for participants to provide terms and the clear instructional prompt was retained. To accommodate both analysis methods, however, each stakeholder was given a large pile of blank cards (in reality 'post-it' notes) as a surrogate for a large sheet of blank paper to imply that many terms were expected in the limited time available. After confirming consent, participants were informed they had five minutes to express every possible project benefit they could think of, noting each on its own card using an individual word. The rear of the cards were marked beforehand so that the researchers could identify the order of term elicitation from each stakeholder. Following standard freelisting practice, the participants were reminded that 'wrong' answers do not exist and that submissions must not be filtered. The imposition of Bousfield and Barclay's time limit (which is absent in more recent elicitation methods) worked together with the emphasis placed on the quantity of terms sought to minimise the risk of term filtering.

In accordance with Bousfield and Barclay's method, participants were not told the cultural domain under study until the instructional prompt was given. This prevented pre-task filtering. The 63 terms elicited from the eight participants (average list length of eight terms) are presented at the end of this paper. They are referred to by number in the following discussion.

\section{Approach A': The Adapted Generalised Protocol}

The adapted generalised protocol ( $A^{\prime}$ ) was tested as follows. Recalling Figure 2, the language of the elicited terms was harmonised to combine terms the analyst considered synonymous. Harmonisation caused five terms to become composites of 16 other terms (Table 1), reducing the collective freelist from 63 terms to 52 . One further term was removed because it was 
duplicated within an individual stakeholder's freelist, again following the generalised freelisting protocol.

\section{TABLE 1 ABOUT HERE}

To prepare the harmonised data for MDS, a similarity matrix was compiled by systematically considering each pair of terms and whether or not the analyst considered them to be related. In accordance with standard approaches to compiling such similarity matrices (Bernard and Ryan

2010), Boolean matrix entries represented the presence or absence of a relationship. Relationship strength or nature was not considered. The analyst's compilation of the similarity matrix was appropriate to the nature of the task at hand: to elicit a meaningful definition of project benefits with sufficient accuracy to inform a business case within the constraint of the limited resourcing found in a typical investment project. As with all codification performed by a single analyst, some bias will be introduced. This can be tackled in two ways: by implementing cross-coder comparison with a team of at least three analysts to identify differing inferences and negotiate consistent interpretation; or by accepting that the simpler, single-analyst process will introduce some bias which, although not desirable, is at least consistent. Although the resulting similarity matrix can inform MDS when configured with the correct parameters, it is of a fundamentally different nature to those used by the generalised protocol. Generalised protocol matrices are compiled arithmetically by calculating the Euclidean distance between each pair of terms on a hypothetical salience construct using the absolute magnitude of the difference between their cognitive salience indices. This produces proximity matrices representing dissimilarity (Leydesdorff and Vaughan 2006). The adapted protocol ( $\left.A^{\prime}\right)$, in contrast, processes a similarity matrix which is a relational rather than a proximity matrix.

The MDS analysis parameters were determined by considering the nature of term similarity. As the matrix represented the analyst's judgement (rather than a measurement) of term associations, non-metric MDS was adopted (Nagpaul, 1999). Further, as the presence of similarity was the exception within the matrix (Figure 3), its presence was more meaningful than its absence. Thus, the Boolean variable in the similarity matrix was asymmetric, with the most important outcome coded as 1 (relationship present) and the other coded as 0 (relationship absent), following convention (SAS Institute Inc., 2008). Because the significance of the value of any pairing within the matrix was asymmetrical, the Lance and Williams agglomerative groupaverage sorting method was used in its binary form, also following convention (Batagelj 1988: 1485, SAS Institute Inc. 2008). 
The similarity matrix was processed using version 18.0 of the PASW software package as discussed above. The MDS analysis had a good goodness of fit (Stress Type I $=0.05867$; Stress Type II = 0.11946; S-Stress $=0.00118$ ). Broad alignment of terms to two dimensional axes was observed. Acknowledging that the dimensional axes illustrating spatial arrangements in the results of MDS "have no meaning [other than to] define a Cartesian coordinate system" (Kenkel and Burchill 2011), the axes were orthogonally rotated by 35 degrees counter-clockwise to produce Figure 4.

\section{FIGURE 4 ABOUT HERE}

It was anticipated that MDS would reveal several clusters of 'like' terms associated by relationships between their constituent pairings of terms (cohesion) and similarity of behaviour with respect to the other terms in the dataset not within the cluster (structural equivalence) (Nagpaul, 1999). Figure 4 demonstrates that this was not the outcome. For this particular dataset, MDS resolved a spatial arrangement of terms centred on their descriptive dimensions alone. As alignment with axes rather than distinct clusters was apparent, terms were ranked by their alignment with each rotated dimension (Tables 2A and 2B). From this, the analyst inferred that one dimension (arbitrarily assigned "Dimension 1") illustrated "Safe access to care" while the other represented "Individuality of care." Possible causes of the assignment of meaning to dimensions rather than clusters is considered in the discussion below.

\section{TABLE 2A ABOUT HERE}

\section{TABLE 2B ABOUT HERE}

\section{Approach B: The Alternative Protocol}

The alternative protocol (B) was tested by continuation of the freelisting elicitation workshop. The workshop adopted a facilitated, participatory format analogous to VM interventions in building projects. Each term remained on its 'post-it' note to provide the cards organised by pilesorting (e.g. (Roos 1998)). The terms were not harmonised prior to analysis (recall Figure 2).

Pilesorting was deployed for two reasons. First, the method reveals the thematic content of a qualitative dataset (Bernard and Ryan 2010) by characterising the cognitive associations between terms held collectively by the individuals from whom the dataset was elicited. This is achieved by manual sorting and grouping (Brewer 1995b, Roos 1998, Trochim and Cabrera 2005, Weller and Romney 2009), which replaces the MDS of the generalised and adapted protocols. Second, the technique is proven in VM workshops to identify thematic concepts from 
brainstorming while building collective understanding. Pilesorting stimulated group 'sensemaking' (Thiry 2001) in the guided form (Maitlis 2005) through which the stakeholders socially constructed common understanding. As the meaning ascribed to clusters of terms was revealed by stakeholder dialogue, the analyst's inferences were not required.

Clusters were identified by the facilitator's direction of stakeholders to negotiate a collective understanding of associated terms by positioning such terms in close proximity to create an affinity diagram. This followed the standard convention of placing all the cards on a table and asking the participants to review and organise the whole dataset into clusters of likeness, with similarity represented by card proximity (Panniers et al. 2003, Blake et al. 2007, Quintiliani et al. 2008). As this approach required the stakeholders to consider all the cards at once, it introduced the risk that some cards would not be adequately considered, harming the development of group consensus. This risk was overcome by the facilitator's introduction of each term in random order and, using the prompt "what is this most similar to?" to ask the stakeholders to negotiate its position in the emerging affinity diagram. Opportunities were created for the reorganisation of the diagram if the stakeholders felt that introduction of a further term revealed a need to relocate, subdivide or combine clusters. A critical aspect of this process is the facilitator's competence in observing, understanding and reacting to the emergent group dynamic. Facilitator action may be required to ensure that dominant actors do not obscure less dominant ones and that less dominant actors are drawn into the discussion to express their views. The suggested used of an accredited VM facilitator should assure this.

The resulting spatial arrangement was reviewed and a consensus opinion that term proximity accurately represented cognitive association secured. Next, the facilitator asked the stakeholders to negotiate a short descriptive name for each cluster that described its constituent terms and the collective understanding ascribed to the cluster by the stakeholders.

Figure 5 presents the resulting affinity diagram (composed from photographs of the working surface used in the workshop). Nine thematically consistent clusters were identified: 'Access (Patient);' 'Benefit to industry;' 'Community;' 'Equipment and environment;,' 'Health / Safety;' 'Healthcare delivery;' 'Patient and staff environment;, 'Strategic;' and 'Sustainable / Environment.'

\section{FIGURE 5 ABOUT HERE}

Cluster constitution (Table 3) varied from one term to 19, with average membership of 7.1. The stakeholders considered the majority of clusters to be thematically consistent, although two were problematic. 'Benefit to industry' was isolated and, on reflection prompted by the facilitator, was not considered sufficiently salient for adoption by the project due to its nature and proposal by a only a single stakeholder. It was removed from the analysis, leaving eight clusters. The 'Healthcare delivery' cluster was exceptionally large, comprising 19 terms. Although the stakeholders stated that those terms were thematically linked, some uncertainty was present. 
Within this cluster, several terms were construed as being associated with the notion of 'privacy' implying the possible presence of a subordinate cluster.

\section{TABLE 3 ABOUT HERE}

Facilitator-prompted exploration of underlying issues in response to discord was required to resolve the 'Healthcare delivery' cluster. On first elicitation, the term 'patient flows' was included. On reviewing proposed content, the proximity of this term to the 'privacy' and 'better privacy (pts)' terms caused stakeholders to identify stronger alignment with terms in the 'Access (patient)' cluster. 'Patient flows' was accordingly moved between clusters.

'Equipment and environment' was considered to clearly communicate the positive outcomes of having access to new equipment. 'Health / Safety' was considered to articulate the need for the infrastructure to enable stakeholders to maintain a hygienic environment; an issue considered distinct from the health and safety issues more commonly associated with building use.

The need for sense-making was greatest for those clusters comprising terms elicited from a small number of stakeholders (Table 4) as these clusters were less salient than those to which more stakeholders contributed. Via discussion, the single stakeholder who contributed $75 \%$ of the terms to the 'Strategic' cluster convinced the other stakeholders of the cluster's validity. Stakeholder discussion of 'Sustainable / Environment' held that, although the cluster was only weakly recognised by those who identified it (with each contributing a single constituent term), it was valid because half of the project stakeholders raised it.

\section{TABLE 4 ABOUT HERE}

Having established consensus regarding term associations and cluster definitions, the stakeholders were directed to conclude the activity. The appropriateness of adopting each cluster of thematically-linked terms as the definition of the project benefits was confirmed. On concluding the alternative protocol (B), the clusters had been ascribed an identity which was an amalgamation of: the terms elicited by freelisting; the clusters of associated terms identified by the affinity diagramming in pilesorting; and the common meaning assigned to clusters by facilitator-led social construction during pilesorting. It was concluded from this understanding that the clusters provided an appropriate definition of project benefits. 


\section{Discussion}

\section{Consequence of Operationalisation for Investment Project Use}

The use of freelisting to elicit common understanding of 'project benefits' has been explored above. As seen, while freelisting can readily elicit cultural domain content, it requires an additional analysis method to expose domain structure. MDS and pilesorting have been evaluated for this role by reviewing their ability to identify sufficiently salient terms in stakeholders' collective cognition for adoption as project benefits for BRMP address.

The adapted freelisting protocol (A') modifies the context of MDS in the generalised freelisting protocol to remove data reduction by salience in acknowledgement of the difficulty of eliciting single-word term expressions in workshop environments. By retaining MDS, the analysis remains largely automated and therefore quick, provided the required software and analytical competency is available. Retention of MDS also provides measures of the 'stress' in the clusters it identifies, allowing illustration validity to be characterised. Indeed, the method may fail to resolve a spatial arrangement of terms within the permitted number of iterations and the two-dimensional layout imposed on all cases to simplify the inference of cluster meaning.

The weaknesses of MDS have, in particular, been exposed by the above analysis. The lack of multiple clusters (in effect two clusters were found with low stress, with each arranged around either notional two-dimensional axis) may have been a feature of the particular dataset or of the stakeholders engaged in the studied case. It is more likely, however, that the absence of the clustering is a shortcoming of the analyst's pairwise inferring of associations between terms when compiling the similarity matrix. This possibility of analyst bias cannot be readily addressed. The team coding used by anthropologists to tackle this issue is unlikely to be viable in building projects due to resource constraints. This issue further suggests that Protocol $A^{\prime}$ is not an appropriate way forward.

The illustration of term associations is formed by MDS independently to stakeholder insight and consensus. This requires meaning to be ascribed to clusters by a separate process. The above has established that the analyst cannot assign meaning without undue bias because they do not share stakeholder understanding and experience. A stakeholder sense-making activity is therefore required. This operationalisation negates a key advantage freelisting when implemented with MDS by the generalised protocol: that stakeholders otherwise need not engage in benefits identification beyond initial term elicitation. As term elicitation could be performed individually and remotely, the potential of MDS to identify benefits without a stakeholder workshop is lost and the attractiveness of the adapted protocol ( $A^{\prime}$ ) to BRMP operators much reduced. Specifically, the opportunity for stakeholders to socially construct shared understanding of associations between elicited terms (and the rationale justifying those associations) by performing group interview tasks such as the affinity diagramming of Protocol $\mathrm{B}$ is lost.

By using pilesorting, the alternative protocol (B) offers the significant advantage of being structured around the stakeholder sense-making that is pivotal to consensus (Maitlis 2005). However, its workshop format causes its effectiveness to be determined by facilitator expertise in managing stakeholder engagement. Despite this, the social construction of common 
understanding of project benefits, makes the pilesorting of the alternative protocol (B) more appropriate for adoption as the method for identifying project benefits. However, its analytical shortcomings and the practical difficulties of convening and facilitating a further stakeholder engagement workshop in the investment process cannot be overlooked. It would seem, therefore, that an ideal approach would combine the rigour of MDS with the social construction of pilesorting.

\section{Address of the Research Questions}

In the address of RQ1, this study has clearly shown that the modified form of MDS used by the adapted protocol ( $A^{\prime}$ ) and the pilesorting of the alternative protocol (B) do not yield similar results. Fundamental differences in the analytical philosophies of the two methods suggest they are dissimilar in intent, outcome and potential effectiveness. Having found different results, consideration of RQ2 does not yield a single answer. Both methods have strengths and, it is suggested, a further, 'ideal' freelisting protocol would combine the most useful elements of both: the analytical rigour of MDS and the social construction of understanding of pilesorting. With the current state of understanding, however, Protocol B most advantageously aligns with sensemaking in construction projects (and the forums available for same such as VM workshops) and is, thus, more compatible with the available skill set. It offers the immediacy of feedback around which stakeholder consensus and buy-in is established. Its effectiveness is, however, dependent on the facilitator's ability to guide stakeholder interactions.

A solution to these practical problems may be found by combining pilesorting with multidimensional scaling. Such 'structured conceptualisation' (see Trochim and Kane 2005, Risisky et al. 2008, and Trochim and Cabrera 2005 for examples) has proven effective when engaging stakeholders in the participatory design of healthcare service delivery pathways. This case has found problems when attempting to use simplified approaches to benefits elicitation when creating healthcare buildings and, in finding that a combination of MDS and pilesorting may also be required this application, has established the case for exploration of structured conceptualisation in this context.

\section{Conclusions}

If freelisting is to identify the project benefits that healthcare buildings must realise, its operationalisation must ensure: parity and continuity of stakeholder engagement; the building of stakeholder consensus; and the creation of an audit trail. While this exploration has established that both MDS and pilesorting can yield insight from freelisting, the social construction of pilesorting avoids the analyst bias that may be introduced when compiling the similarity matrix required by MDS. Moreover, pilesorting has been found to flow directly from term elicitation as both can be deployed in the same workshop. Assuming a competent facilitator, pilesorting gives every stakeholder the opportunity to comprehend others' contributions so that a satisficing definition of project benefits can be negotiated from an informed position. MDS' disengagement from stakeholders does not facilitate this social construction of common understanding.

Overall, the above suggests that the linking of freelisting (as the term elicitation process) to pilesorting (as the project benefit identification process) would provide a project benefits elicitation method. However, pilesorting has two weaknesses. First, its efficacy depends entirely 
on the facilitator's understanding of the technique and competency in ensuring parity of stakeholder engagement. Second, it does not readily provide an audit trail. Although the project benefits identified by the elicitation workshop will be documented (as will the freelisted terms within the definition of each), the discussions held in agreeing those definitions are less readily recorded. As the understanding of each project benefit is determined by the terms within it and the discussion by which their association was negotiated, it cannot be fully expressed in documented form. This drawback is also the main advantage of pilesorting: that the social construction of common understanding is stimulated.

It is accordingly recommended that further work addresses the development of a project benefits elicitation method largely based on the association of freelisting with pilesorting, but augmented with the additional rigour of either Weller's consensus modelling (with the attendant failings of its non-participatory approach) or the emergent field of structured conceptualisation. 


\section{Index of Terms}

The elicited terms are illustrated as below in the Figures. See Table 1 for the content of the composite terms 1 to 5 . Note that words identifying the studied project have been redacted.

1: $\quad$ new and modern building [CompA]

2: $\quad$ sustainable and energy efficient [CompD]

3: $\quad$ patient privacy [CompC]

4: $\quad$ cleanliness [CompB]

5: $\quad$ purpose built [CompE]

6: $\quad$ new equipment

7: infection control

8: $\quad$ patients on same site for tests

9: $\quad$ single rooms-con-IC-peace/O

10: access to xray and other facilities

11: staff care (including night)

12: $\quad$ convenient for transport

13: better environment patient and staff

14: improvement in activity

15: happy workforce

16: $\quad$ upholds dignity (pts)

17: $\quad$ safe access

18: reduction in complaints

19: equality of access to services

20: improvement in health care

21: easy to maintain

22: $\quad$ aesthetically pleasing

23: user friendly

24: community focal point

25: $\quad$ service adjacencies

26: in the hospital 'community'

27: patient flows

28: detail design

29: $\quad$ perception and awareness

30: delivering on promises ([redacted])

31: individual care

32: quality environment

33: $\quad$ space

34: outlook

35: well equipped

36: public consultation

37: safe

38: bright

39: welcome

40: $\quad$ supportive

41: easy access

42: friendly

43: improved facilities for patients

44: improved facilities for staff

45: $\quad$ garden space for patients

46: $\quad$ improving [redacted] site

47: boost for construction industry/more work

48: improved transport to [redacted]

49: better facilities for patients

50: better facilities for staff

51: patient safety

52: $\quad$ single rooms 


\section{References}

Abdul-Samad, Z., \& Macmillan, S. (2005) Improving Design Quality and Value in the Built Environment through Knowledge of Intangibles. International Engineering Management Conference, 0-7803-8519-5/04, 2004 IEEE International, 18-21 Oct., pp. 898-902.

Ares, G., \& Deliza, R. (2010) Identifying important package features of milk desserts using free listing and word association. Food Quality and Preference, 21(6), 621-628.

Batagelj, V. (1988) Generalized Ward and Related Clustering Problems. in Bock, H.H. (ed.) Classification and Related Methods of Data Analysis. Amsterdam: North-Holland, pp. 67-74..

Bernard, H.R. (2006) Research Methods in Anthropology: Qualitative and Quantitative Approaches (Fourth Edition). Oxford: Altamira.

Bernard, H.R., \& Ryan, G.W. (2010) Analyzing Qualitative Data. Thousand Oaks, CA: SAGE Publications.

Blake, C.E., et al. (2007) Classifying foods in contexts: How adults categorize foods for different eating settings. Appetite, 49(2), 500-510.

Block, M.A.G., et al. (2001) Beyond health gain: the range of health system benefits expressed by social groups in Mexico and Central America. Social Science \& Medicine, 52, 1537-1550.

Bousfield, W., \& Barclay, W. (1950) The Relationship between Order and Frequency of Occurrence of Restricted Associative Responses. Journal of Experimental Psychology, 40(5), 643-647.

Brewer, D. (1995a) Patterns in the Recall of Persons in a Department of a Formal Organization. Journal of Quantitative Anthropology, 5(4), 255-284.

Brewer, D. (1995b) Cognitive Indicators of Knowledge in Semantic Domains. Journal of Quantitative Anthropology, 5(2), 107-128.

CABE (2006) Buildings and spaces: why design matters. London: Commission for Architecture and the Built Environment.

Codinhoto, R. et al., 2010. The Effects of the Built Environment on Health Outcomes. Salford: School of the Built Environment Research Institute for the Built and Human Environment.

Department for Culture, Media and Sport (2000) Better Public Buildings: A proud legacy for the future. London: HM Government.

Ensign, J., \& Gittelsohn, J. (1998) Health and Access to Care: Perspectives of Homeless Youth in Baltimore City, U.S.A. Soc. Sci. Med., 47(12), 2087-2099.

Fleisher, M.S., \& Harrington, J.A. (2009) Freelisting: Management at a Women's Federal Prison Camp. in de Munchk, V.C. \& Sobo, E.J. (eds), Using Methods in the Field: A Practical Introduction and Case Book. Walnut Creek, CA: AltaMira Press, pp. 69-84.

Gann, D., Salter, A., \& Whyte, J. (2003) Design Quality Indicator as a tool for thinking. Building Research and Information, 31(5), 318-333.

Hamilton, A. (2002) Considering value during early project development: a product case study. International Journal of Project Management, 20, 131-136. 
HM Treasury (2003) The Green Book: Appraisal and Evaluation in Central Government. London: TSO.

Jenkins, P., \& Forsyth, L. (Eds.) (2010) Architecture, Participation and Society. Abingdon: Routledge.

Kenkel, N.C., \& Burchill, C.E. (2011) Rigid rotation of nonmetric multidimensional scaling axes to environmental congruence. Abstractia Botanica, 14, 109-110.

Leydesdorff, L., \& Vaughan, L. (2006) Co-occurrence matrices and their applications in information science: Extending ACA to the Web environment. Journal of the American Society for Information Science and Technology, 57(12), 1616-1628.

Luck, R. (2010) Learning to talk to users in participatory design situations. Design Studies, 28, 217-242.

Maitlis, S. (2005) The Social Processes of Organizational Sensemaking. The Academy of Management Journal, 48(1), 21-49.

McIntyre, D., \& Ataguba, J.E. (2011) How to do (or not to do) ... a benefit incidence analysis. Health Policy and Planning, 26(2), 174-182.

Mills, G. et al. (2011) Review of Evidence for the Contribution of Healthcare Infrastructure Investment to Health Gain, with Particular Reference to Hospitals ( $\mathrm{HIIHG)}$. Health and Care Infrastructure Research and Innovation Centre; European Centre for Health Assets and Architecture.

Morris, P., \& Jamieson, A. (2004) Linking Corporate Strategy To Project Strategy via Portfolio and Program Management. In Proceedings of EURAM 04, St Andrews.

MSP, \& Office of Government Commerce (2007) Managing Successful Programmes. London: The Stationary Office.

Nagpaul, P.S. (1999) Multidimensional Scaling. In Guide to Advanced Data Analysis using IDAMS Software. Available from: http://www.unesco.org/webworld/idams/advguide/TOC.htm [Last accessed: 6 June 2011]: UNESCO Secretariat.

Panniers, T.L., Feuerbach, R.D., \& Soeken, K.L. (2003) Methods in informatics: using data derived from a systematic review of health care texts to develop a concept map for use in the neonatal intensive care setting. Journal of Biomedical Informatics, 36(4-5), 232-239.

Pearce, D., \& Özdemiroglu, E. (2002) Economic Valuation with Stated Preference Techniques: Summary guide. London: Department for Transport, Local Government and the Regions, ISBN: 1851125698.

Quintiliani, L.M. et al. (2008) The Use of the Pile Sort Method in Identifying Groups of Healthful Lifestyle Behaviors among Female Community College Students. Journal of the American Dietetic Association, 108(9), 1503-1507.

Ratneshwar, S., et al. (1997) Benefit salience and consumers' selective attention to product features. International Journal of Research in Marketing, 14, 245-259.

Reiss, G. et al. (2006) The Gower Handbook of Programme Management. Aldershot: Gower Publishing. 
Remenyi, D., \& Sherwood-Smith, M. (1998) Business benefits from information systems through an active benefits realisation programme. International Journal of Project Management, 16 (2), 81-98.

Risisky, D., et al. (2008) Concept Mapping as a Tool to Engage a Community in Health Disparity Identification. Ethnicity \& Disease, 18(1), 77-83.

Rokeach, M. (1973) The Nature of Human Values. New York: The Free Press.

Rooke, J.A., et al. (2010) Lean Knowledge Management: The Problem of Value. in Proceedings 18th International Conference of the International Group for Lean Construction, IGLC-18. Technion, Haifa, Israel, 14-16 July, pp. 12-21.

Roos, G. (1998) Pile Sorting: "Kids Like Candy." in de Munchk, V.C. \& Sobo, E.J. (eds), Using Methods in the Field: A Practical Introduction and Case Book. Walnut Creek, CA: AltaMira Press, pp. 97-110.

Sapountzis, S., et al. (2009) Realising benefits in primary healthcare infrastructures. Facilities, 27 $(3 / 4), 74-87$.

SAS Institute Inc. (2008) The DISTANCE Procedure. In SAS/STAT 9.2 User's Guide . Cary, NC: SAS Institute Inc., pp. 1486-1531.

Schmitt, N. (1998) Quantifying word association responses: what is native-like? System, 26, 389-401.

Schrauf, R.W., \& Sanchez, J. (2008) Using Freelisting to Identify, Assess, and Characterize Age Differences in Shared Cultural Domains. Journal of Gerontology: Social Sciences, 63B(6), S385-S393.

Schrauf, R.W. \& Sanchez, J. (2010) Age Effects and Sample Size in Free Listing. Field Methods, 22 (1), 70-87.

Simon, H.A. (1957) Models of Man: Social and Rational: Mathematical Essays on Rational Human Behavior in a Social Setting. New York: Wiley.

Smith, J.J. (1993) Using ANTHOPAC 3.5 and a Spreadsheet to Compute a Free-List Salience Index. Cultural Anthropology Methods, 5(3), 1-3.

Smith, J.J., \& Borgatti, S.P. (1997) Salience Counts - And So Does Accuracy: Correcting and Updating a Measure for Free-List-Item Salience. Journal of Linguistic Anthropology, 7(2), 208-209.

Sutrop, U. (2001) List Task and a Cognitive Salience Index. Field Methods, 13(3), 263-276.

Sweeney, J.C., \& Soutar, G.N. (2001) Consumer perceived value: The development of a multiple item scale. Journal of Retailing, 77, 203-220.

The Scottish Government (2009a) Scottish Capital Investment Manual: Project Evaluation Guide. Edinburgh: The Scottish Government.

The Scottish Government (2009b) Scottish Capital Investment Manual: Supporting Guidance: Design Assessment in the Business Case Process. Edinburgh: The Scottish Government. 
Thiry, M. (2001) Sensemaking in value management practice. International Journal of Project Management, 19(2), 71-77.

Thomson, D., et al. (2010) "Quantifying the Benefits of Healthcare Infrastructure Investment." in Proceedings, HaCIRIC International Conference 2010. Edinburgh, pp. 57-74.

Thomson, D.S., et al. (2003) Managing value and quality in design. Building Research and Information, 31(5), 334-345.

Trochim, W., \& Kane, M. (2005) Concept mapping: an introduction to structured conceptualization in health care. International Journal for Quality in Health Care, 17(3), 187.

Trochim, W.M., \& Cabrera, D. (2005) The complexity of concept mapping for policy analysis. Emergence: Complexity and Organization, 7(1), 11-22.

Ulrich, R.S. et al. (2008) A Review of the Research Literature on Evidence-Based Healthcare Design. Georgia Tech College of Architecture; The Center for Healthcare Design.

Weller, S.C. (2007) Cultural Consensus Theory: Applications and Frequently Asked Questions. Field Methods, 19(4), 339-368.

Weller, S.C., \& Romney, A.K. (2009) Systematic Data Collection. Qualitative Research Methods, Volume 10. Newbury Park, California: Sage Publications. 


\begin{tabular}{|c|c|c|c|}
\hline $\begin{array}{l}\text { Elicit Terms } \\
\text { Freelisting }\end{array}$ & $\begin{array}{c}\text { Harmonise Terms } \\
\text { Codification }\end{array}$ & $\begin{array}{c}\text { Reduce Data } \\
\text { Cognitive salience indices } \\
\text { and scree plot }\end{array}$ & $\begin{array}{l}\text { Cluster Analysis } \\
\text { Multidimensional scaling of } \\
\text { Euclidean distance matrix }\end{array}$ \\
\hline
\end{tabular}

FIGURE 1: The Generalised Freelisting Protocol

(A)

\begin{tabular}{|c|c|c|c|}
\hline $\begin{array}{l}\text { Elicit Terms } \\
\text { Freelisting }\end{array}$ & $\begin{array}{c}\text { Harmonise Terms } \\
\text { Codification }\end{array}$ & $\begin{array}{c}\text { Reduce Data } \\
\text { Cognitive salience indices } \\
\text { and scree plot }\end{array}$ & $\begin{array}{l}\text { Cluster Analysis } \\
\text { Multidimensional scaling of } \\
\text { Euclidean distance matrix }\end{array}$ \\
\hline (Stakeholders) & (Analyst) & (Analyst) & (Analyst) \\
\hline
\end{tabular}

$\left(A^{\prime}\right)$

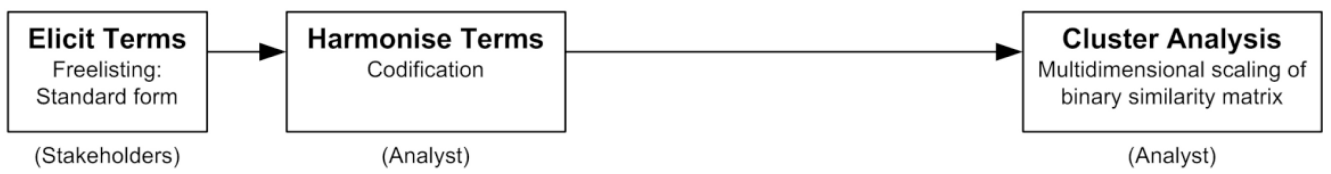

(B)

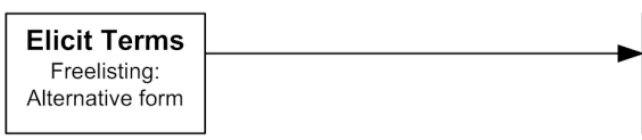

Pilesorting

Data reduction and clustering by facilitated negotiation

(Stakeholders)

(Stakeholders)

FIGURE 2: The Generalised (A), Adapted Generalised (A'), and Alternative (B) Protocols for Freelisting Common Understanding of Benefits Sought from Healthcare Buildings 


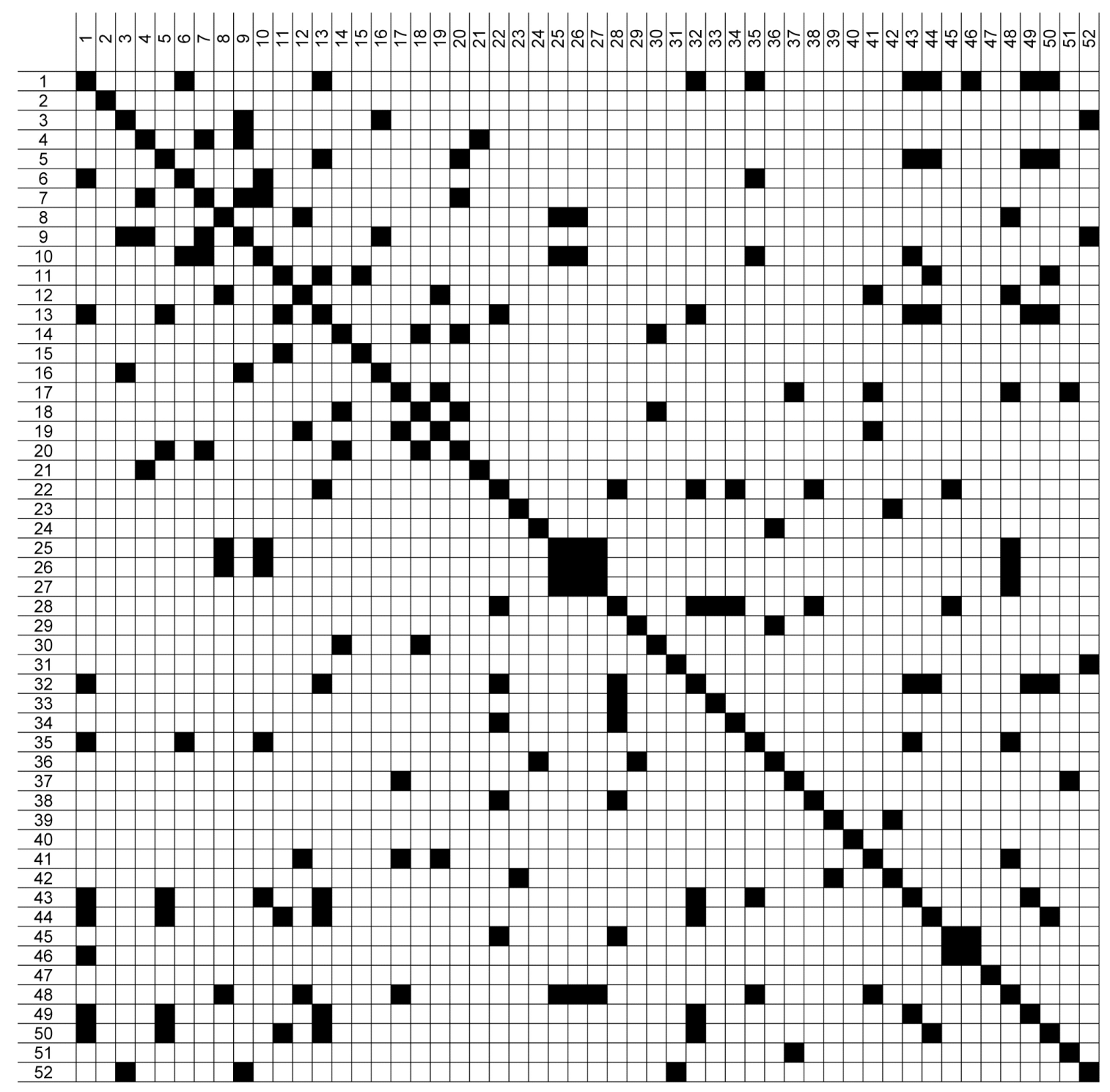

FIGURE 3: Similarity Matrix of Harmonised Freelist Terms 


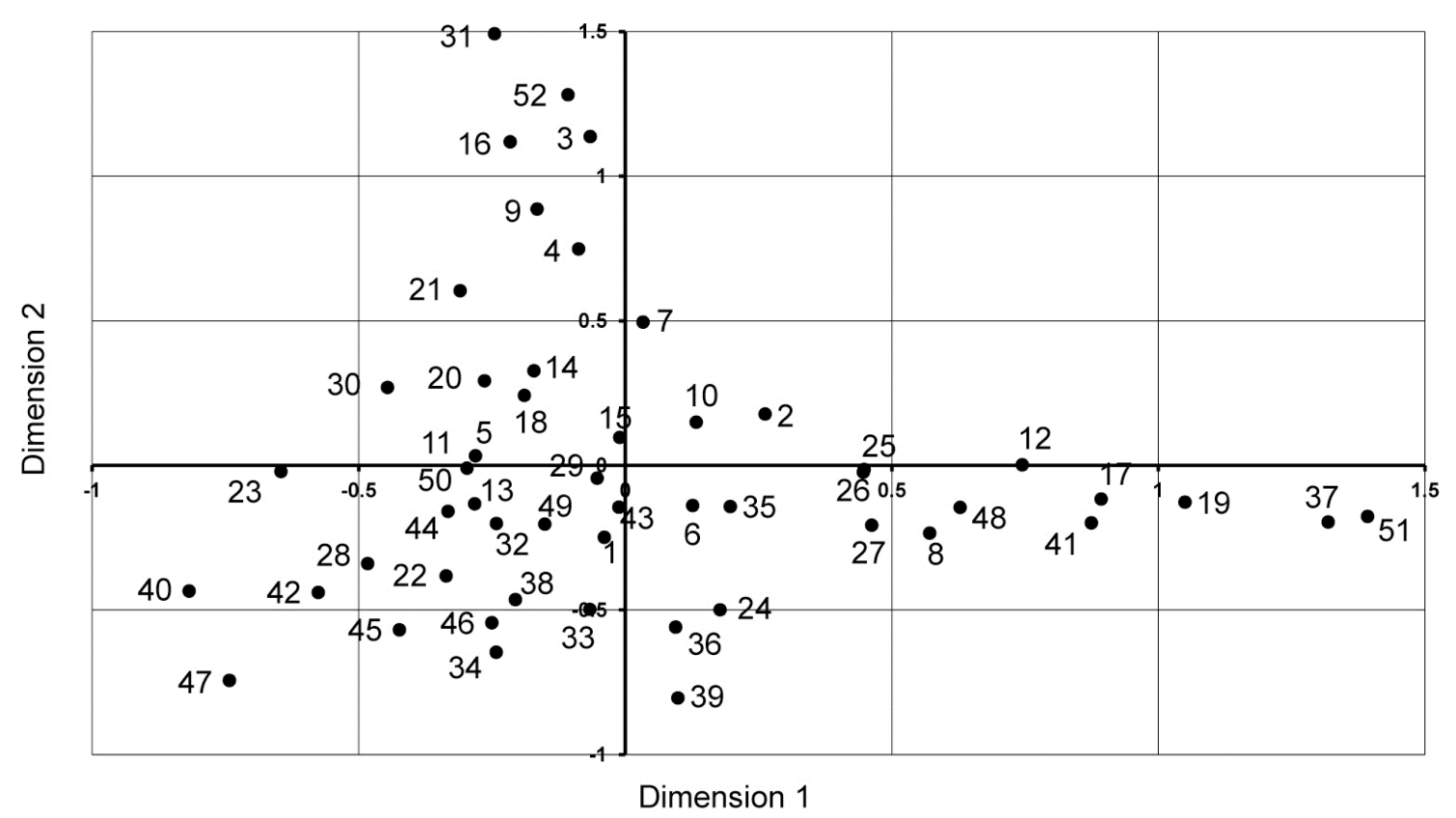

FIGURE 4: Rotated Clustering of 'Like' Terms resulting from MDS under the Adapted Protocol ( $\left.A^{\prime}\right)$

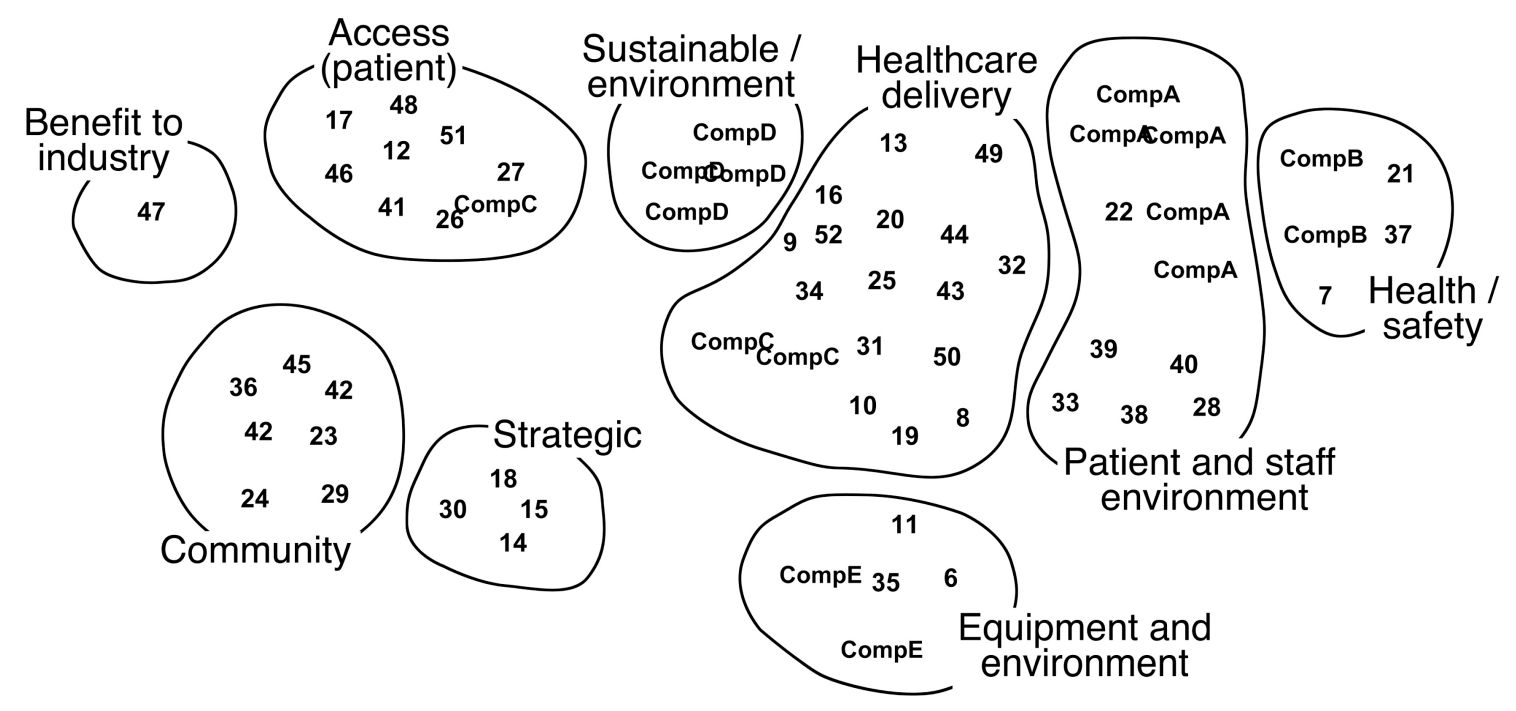

FIGURE 5: Affinity Diagram Resulting from Pilesorting Activity 
Composite Term

CompA: New and modern building

\section{Constituent Terms}

A3: Modern building (heating etc.)

C4: Modern facility

D1: New building

H1: Lovely new build

H5: Modern building

\begin{tabular}{lll}
\hline CompB: & Cleanliness & B2: Easy to clean \\
& & E2: Clean \\
\hline CompC: & Patient privacy & B3: Better privacy (pts) \\
& & D2: Privacy \\
& & F2: Privacy for patients \\
\hline CompD: & Sustainable and & B5: Sustainable efficiency building \\
& energy efficient building & C1: Sustainable \\
& & D12: Energy efficient \\
& & G2: Energy efficient building \\
\hline CompE: & Purpose built & D8: Bespoke design \\
& & H7: Gym layout as we would like \\
\hline
\end{tabular}

TABLE 1: Composite Terms Introduced to Harmonise Language 


\begin{tabular}{ccc}
$\begin{array}{c}\text { Dimension 1 } \\
\text { Alignment }\end{array}$ & $\begin{array}{c}\text { Dimension 2 } \\
\text { Alignment }\end{array}$ & Term \\
\hline 1.393 & -0.177 & 51: patient safety \\
\hline 1.318 & -0.195 & 37: safe \\
\hline 1.05 & -0.127 & $19:$ equality of access to services \\
\hline 0.893 & -0.116 & 17: safe access \\
\hline 0.874 & -0.198 & $12:$ convenient for transport \\
\hline 0.745 & 0.002 & $48:$ improved transport to [redacted] \\
\hline 0.628 & -0.146 & $8:$ patients on same site for tests \\
\hline 0.571 & -0.234 & 27: patient flows \\
\hline 0.462 & -0.206 & $25:$ service adjacencies \\
\hline 0.448 & -0.014 &
\end{tabular}

TABLE 2A: Alignment of Terms with Dimension 1: "Safe Access to Care"

\begin{tabular}{ccc}
$\begin{array}{c}\text { Dimension 1 } \\
\text { Alignment }\end{array}$ & $\begin{array}{c}\text { Dimension 2 } \\
\text { Alignment }\end{array}$ & Term \\
\hline-0.245 & 1.493 & 31: individual care \\
\hline-0.107 & 1.281 & 52: single rooms \\
\hline-0.066 & 1.137 & 3: patient privacy \\
\hline-0.216 & 1.119 & 16: upholds dignity (pts) \\
\hline-0.166 & 0.886 & 4: cleanliness \\
\hline-0.087 & 0.749 & 21: easy to maintain \\
\hline-0.31 & 0.604 & $14:$ improction control \\
\hline 0.033 & 0.496 & $20:$ improvement in activity \\
\hline-0.171 & 0.327 &
\end{tabular}

TABLE 2B: Alignment of Terms with Dimension 2: "Individuality of Care" 


\section{Access (patient)}

safe access

improving [redacted] site

easy access

convenient for transport

improved transport to [redacted]

in the hospital 'community'

patient safety

patient flows

\section{Healthcare Delivery}

upholds dignity (pts)

better environment patient and staff

better facilities for staff

quality environment

improvement in health care

single rooms

single rooms-con-IC-peace/Q

service adjacencies

individual care

access to xray and other facilities

privacy for patients

better privacy (pts)

equality of access to services

MOE patients on same site for

tests

improved facilities for staff

improved facilities for patients

better facilities for patients

privacy

outlook

\section{Benefit to Industry}

boost for construction

industry/more work

\section{Community}

public consultation

friendly

friendly

user friendly

perception and awareness

community focal point

garden space for patients

Patient and Staff Environment

modern building

lovely new build

aesthetically pleasing

modern building (heating etc)

modern facility

new building

welcome

supportive

bright

detail design

space
Equipment and Environment

gym layout as we would like

well equipped

new equipment

staff care (including night)

bespoke design

\section{Health / Safety}

safe

clean

easy to clean

easy to maintain

infection control

\section{Strategic}

delivering on promises

([redacted])

improvement in activity

happy workforce

reduction in complaints

\section{Sustainable / Environment}

sustainable

energy efficient building

energy efficient

sustainable efficiency building

TABLE 3: Clustered Terms Grouped by Stakeholder-Inferred Project Benefit Names 


\begin{tabular}{|c|c|c|c|c|c|c|c|c|}
\hline \multirow[b]{2}{*}{ Respondent } & \multicolumn{8}{|c|}{ Cluster } \\
\hline & 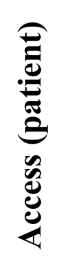 & ن & 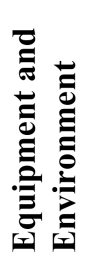 & 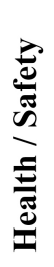 & 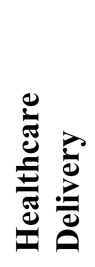 & 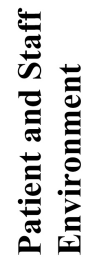 & 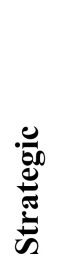 & 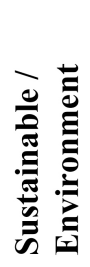 \\
\hline $\mathbf{A}$ & 1 & - & 1 & - & 1 & 1 & - & - \\
\hline B & 1 & - & - & 1 & 5 & - & 3 & 1 \\
\hline $\mathbf{C}$ & 1 & 2 & - & 1 & 1 & 2 & - & 1 \\
\hline D & - & 2 & 2 & - & 4 & 3 & 1 & 1 \\
\hline $\mathbf{E}$ & 1 & 2 & - & 2 & 1 & 2 & - & - \\
\hline $\mathbf{F}$ & 2 & 1 & - & - & 3 & - & - & - \\
\hline $\mathbf{G}$ & 2 & - & - & - & 2 & 1 & - & 1 \\
\hline $\mathbf{H}$ & - & - & 2 & 1 & 2 & 2 & - & - \\
\hline Term Count & 8 & 7 & 5 & 5 & 19 & 11 & 4 & 4 \\
\hline
\end{tabular}

TABLE 4: Distribution of Cluster Terms by Participant 